\title{
Médiévales
}

Langues, Textes, Histoire

$64 \mid$ printemps 2013

Temporalités de l'Égypte

\section{Les aḥbās de Fusțāt aux deux premiers siècles de l'Hégire : entre pratiques socio-économiques et normalisation juridique}

Between Socio-economic Practices and Legal Normalization: The ahbās of Fusțāt during the First Two Centuries of Hegira

\section{Sobhi Bouderbala}

\section{OpenEdition \\ Journals}

Édition électronique

URL : https://journals.openedition.org/medievales/6937

DOI : 10.4000/medievales.6937

ISSN : $1777-5892$

\section{Éditeur}

Presses universitaires de Vincennes

\section{Édition imprimée}

Date de publication : 1 juillet 2013

Pagination : $37-55$

ISBN : 978-2-84292-371-6

ISSN : 0751-2708

\section{Référence électronique}

Sobhi Bouderbala, «Les ahbās de Fustāt aux deux premiers siècles de l'Hégire : entre pratiques socioéconomiques et normalisation juridique », Médiévales [En ligne], 64 I printemps 2013, mis en ligne le 07 septembre 2013, consulté le 23 avril 2022. URL : http://journals.openedition.org/medievales/6937 ; DOI : https://doi.org/10.4000/medievales.6937 
Médiévales 64,printemps 2013, p. 37-56

Sobhi BOUDERBALA

\section{LES AHBĀS DE FUSṬĀṬ AUX DEUX PREMIERS SIÈCLES DE L'HÉGIRE : ENTRE PRATIQUES SOCIO-ÉCONOMIQUES ET NORMALISATION JURIDIQUE}

L'institution des biens de mainmorte en Islam, le $w_{a q f}{ }^{1}$, a fait l'objet de nombreuses études, ce qui n'a rien d'étonnant vu son importance dans l'histoire économique, sociale et juridique de l'Islam. Des traités, ainsi que des documents de waqf, permettent aujourd'hui de comprendre assez bien le fonctionnement de cette institution, ainsi que son évolution ${ }^{2}$. En revanche, toute une phase de l'histoire du waqf - la période fondatrice, celle des origines - reste à ce jour très mal connue parce que très peu étudiée. Trois études ont été consacrées à la genèse de cette institution au début de l'Islam ${ }^{3}$, mais sans mettre suffisamment l'accent sur son essence ni sur l'évolution historique qu'elle a connue jusqu'à

1. Le terme waqf, substantif de waqqafa et awqafa, veut dire littéralement immobilisation, il désigne classiquement les biens de mainmorte, inaliénables en Islam. Le terme $a h b \bar{a} s$, sing, $h u b s$, choisi pour le titre de cet article, désigne aussi la même institution, mais il est plus ancien que le terme de waqf. Et c'est le terme utilisé en Égypte tout au long de la période étudiée comme nous l'expliquerons plus loin. Pour une définition générale du waqf, avec en toile de fond la question des origines de cette institution, voir P. Hennigan, The Birth of a Legal Institution. The Formation of the waqf in Third-Century A.H. Hanafi Legal Discourse, Leyde, 2004, p. 50-70.

2. La bibliographie sur l'institution du waqf est immense. Nous nous limiterons à signaler les principaux corpus documentaires des waqf-s, concernant Le Caire et Jérusalem. Voir S. Denoix, «Pour une exploitation d'ensemble d'un corpus: les waqfs mamelouks du Caire», dans R. DeGuilHEM (éd.), Le Waqf dans l'espace islamique: outil de pouvoir socio-politique, Damas, 1995, p. 29-44 (Le Caire mamelouk); D. P. LitTLE, A Catalogue of the Islamic Documents from al-Haram al-Šarif in Jerusalem, Wiesbaden, 1984 (Jérusalem mamelouke).

3. J. Sснаснт, «Early Doctrines of Waqf», dans Mélanges Fuad Köprülü, Istanbul, 1953, p. 443-452; Cl. CAHEN, «Réflexions sur le waqf ancien», Studia Islamica, 14 (1961), p. 37-56; M. GIL, «The Earliest Waqf Foundations», Journal of the Near Eastern Studies, 57/2 (1998), p. 125-140. Si l'on excepte l'étude de M. Gil, qui s'intéresse aux fondations aumônières (șadaqāt) du Prophète et de ses compagnons, les deux autres travaux se fondent exclusivement sur des ouvrages juridiques, 
sa normalisation juridique à la fin $\mathrm{du} \mathrm{II} / \mathrm{VIII}{ }^{\mathrm{e}}$ siècle et au début du $\mathrm{III}^{\mathrm{e}} / \mathrm{IX}^{\mathrm{e}}$ siècle . Rien d'étonnant à cela: l'on manque cruellement d'informations sur ces biens de mainmorte pendant cette période fondatrice, sur leur fonctionnement dans la pratique et sur les différents problèmes auxquels les premiers cadis étaient confrontés dans la gestion de fondations qui étaient alors quasi exclusivement privées (waqf ahlī).

Dans ce contexte, la littérature pré-fatimide de Fusțāt fait figure d'exception. De nombreuses informations y sont livrées quant à la gestion de ces biens par les premiers cadis de la ville. Surtout, on dispose de quelques spécimens - au nombre de quatre - de waqf-s primitifs remontant à l'époque umayyade et intégrés dans les ouvrages des auteurs fustạțiens pré-fatimides ${ }^{4}$. Jumelés avec deux documents sur papyrus remontant respectivement au début $\mathrm{du} \mathrm{II}^{\mathrm{e}} / \mathrm{VIII}^{\mathrm{e}}{ }^{5}$ et au début $\mathrm{du} \mathrm{III/} / \mathrm{IX}^{\mathrm{e}}$ siècle ${ }^{6}$, ils forment un petit corpus unique, susceptible de nous éclairer sur les pratiques socio-économiques relatives à ces biens, puis leur normalisation par les écoles juridiques mālikites et ḥanafites, les deux madhab-s dominants en Égypte dans la deuxième moitié $\mathrm{du} \mathrm{II} / \mathrm{VIII}^{\mathrm{e}}$ siècle $^{7}$.

Une remarque préalable: le terme utilisé dans la littérature pré-fatimide pour désigner ces biens de mainmorte anciens de Fusțât est celui de hubs. Un autre terme se confond avec le h̆ubs, c'est le vocable șadaqa, aumône. On parle souvent de hubs șadaqa, dans le sens de bien de mainmorte à visée charitable ${ }^{8}$. Le

essentiellement les deux traités hananafites rédigés par des Irakiens au début du $\mathrm{III}^{\mathrm{e}} / \mathrm{IX}^{\mathrm{e}}$ siècle: HILĀL AL-RAY', Ahkām al-waqf, Hyderabad, 1936; AL-HूAssĀF, Ahkam al-awqāf, Le Caire, 1902.

4. IBN 'ABd AL-HaKAm, Futūh Mișr wa ahbāruhā, éd. C. ToRrey, New Haven, 1923; AL-Kindī, Kitāb al-wulāt wa kitāb al-qudāt, éd. R. Guest, Londres, 1912; IBN Yūnus, Ta'rīh Ibn Yūnus, 2 vols, éd. A. Fathī, Beyrouth, 2000. Des ouvrages de cette période - notamment ceux d'al-Kindī sont aujourd'hui perdus, mais ils ont dû circuler au moins jusqu'à la conquête ottomane d'Égypte en 1517 puisque les grands polygraphes mamelouks en avaient des copies dont des passages entiers sont insérés dans leurs œuvres. Nous en donnerons quelques exemples dans cette étude.

5. A. Hanafi, «An Arabic Will Written on a Chip», dans G. Traianos éd., Proceedings of the Twenty-Fifth International Congress of Papyrology, Ann Harbor 2007, Ann Harbor, 2010, p. 299306 (= P. Ragab 6).

6. Y. RaGiB, Marchands d'étoffes du Fayyoum au III /IXe siècle d'après leurs archives (actes et lettres), vol. I, Les actes des Ban̄ 'Abd al-Mu'min, Le Caire, 1982, p. 36-45 (= P. Marchands I $12)$.

7. M. Tillier (trad.), Vies des cadis de Mișr 237/851-366/976. Extrait du Raf' al-ișr 'an qud̄āt Mișr d'Ibn Hağar al- 'Asqalān̄, Le Caire, 2002, p. 16-18.

8. SAHNŌN B. SA 'īD, al-Mudawwana al-kubrā, Beyrouth, 1994, p. 417 (titre du chapitre: al-hubs wa-l-șadaqa). Voir les analyses attribuées à Mālik concernant l'association des deux termes et les problèmes juridiques relatifs à leur utilisation dans les pages 419-421. L'appellation de ces biens inaliénables par les deux termes jumelés hubs șadaqa semble avoir perduré au moins jusqu'au début de l'époque fatimide en Égypte, comme le montre l'acte de hubs daté de 400/1009 inclus dans l'ouvrage d'Ibn 'Abd al-Zāhir, al-Rawḍa al-bahiyya al-zāhira fì hiṭtạ al-Mu'uziyya al-Qāhira, éd. A. F. SAYYID, Le Caire, 1996, p. 145. 
terme waqf n'est jamais utilisé dans la documentation égyptienne de l'époque qui nous intéresse. La question est d'importance car, comme l'a récemment montré Peter Hennigan, il s'agissait pour les deux théoriciens ḥanafites sur lesquels l'on s'appuie généralement - Hilāl et al-Hașṣāf - d'inventer un véritable statut juridique, en imposant le terme de waqf à l'institution des biens de mainmorte, et de la différencier des autres catégories de propriétés ${ }^{9}$. D'où l'intérêt de cette documentation égyptienne primitive qui se place chronologiquement dans un cadre juridique marqué par l'absence de toute doctrine sur l'institution, un cadre marqué par la coutume (le 'urf) sur laquelle nous n'avons pas d'informations précises, par les pratiques sociales qui se manifestent dans les clauses mêmes des quelques spécimens exhumés, mais aussi par des pratiques juridiques locales, œuvres des jurisconsultes de la ville ${ }^{10}$.

Un récit bien connu, rapporté par al-Kindī, donne un aperçu de l'état des biens inaliénables à Fusțâț au début du II/VIII ${ }^{\mathrm{e}}$ siècle, et de la volonté de l'appareil judiciaire de s'en approprier la gestion ${ }^{11}$ :

Le premier cadi à mettre la main sur les biens de mainmorte ( $a h b \bar{a} s)$ à Miṣr fut Tawba b. Namir, sous le règne de Hišām. [Jusque-là], les biens de mainmorte étaient seulement gérés par leurs bénéficiaires $(a h l i-h \bar{a})$ et par les exécuteurs testamentaires (awșiya $\bar{a}^{\prime}$ ) [des fondateurs]. Quand Tawba b. Namir fut [cadi], il dit: «Je considère que les pauvres et les indigents sont les ultimes bénéficiaires des revenus de ces aumônes (sadaqāt): je suis donc d'avis de prendre en main leur gestion afin de les préserver de la ruine et d'empêcher qu'ils ne soient transmis à des héritiers lointains... Le premier cadi de Mișr aux archives (dīwān) duquel les biens de mainmorte ( $a h h b \bar{a} s)$ furent versés fut Tawba b. Namir, en 118/736.

La décision de Tawba est précieuse à plusieurs égards. Outre le fait qu'elle nous renseigne sur le caractère privé de ces fondations (qui seront appelées plus tard $a h l \bar{\imath}$ ), elle montre probablement que la règle juridique utilisée à Fusțāt (et sans doute ailleurs) était d'immobiliser un bien au profit de sa descendance, mais aussi de désigner les pauvres et les indigents comme ultimes bénéficiaires de l'usufruit de ces biens. Dans cet extrait, la décision du cadi laisse transparaître des cas de contournement ou d'abus de la part des bénéficiaires ou des exécuteurs

9. P. Hennigan, The Birth of a Legal Institution..., p. 70. Sur les significations des différents termes composant le champ sémantique de l'institution en question (hubs, șadaqa, waqf), voir p. 71-81.

10. Cela pose - à nouveau - la question des «écoles» juridiques proposées par J. SсHACHT, Introduction au droit musulman, traduit de l'anglais par P. Kempf et A. Turki, Paris, 1983, p. 35-40. Pour une analyse détaillée de l'école égyptienne, voir M. TiLLIER, «Les "premiers" cadis de Fusțāt et les dynamiques régionales de l'innovation judiciaire», Annales Islamologiques, 45 (2011), p. 214218.

11. Al-KIndī, Kitāb al-wulāt..., p. 346; trad. M. Tillier, Histoire des cadis égyptiens. Ahbār qud̄āt Miṣr, Le Caire, 2012, p. 102 (traduction modifiée). 
testamentaires. Elle révèle aussi la volonté du pouvoir judiciaire d'intégrer en son sein un patrimoine financier et foncier important de la ville en en assurant directement la gestion. Cela nous conduira à étudier les quelques traces de biens inaliénables de Fusțāt conservées par la littérature pré-fatimide pour mieux comprendre leur fonctionnement et la structure de leurs actes d'immobilisation ; ainsi pourrons-nous nous interroger sur les continuités et les ruptures entre les pratiques sociales en matière de hubs et les normes juridiques en vigueur à Fusțâtt.

Le premier spécimen d'un acte de hubs est livré par Ibn 'Abd al-Hakam (m. 271/871) : il s'agit d'une demeure célèbre à Fusțâț, la dār al-silsila ${ }^{12}$. Originellement concession foncière de Abū 'Abd al-Raḥmān al-Fihrī, membre de l'armée de conquête, elle devint la propriété de son petit-fils, al-Hārit b. 'Alā', qui la constitua en bien inaliénable. Voici en quels termes Ibn 'Abd al-Hakam décrit la procédure d'immobilisation de la dār al-silsila:

Il (i.e e. al-Hāriț constitua la dār [al-silsila] bien de mainmorte au profit des plus proches descendants $\left(a l-a q^{\prime} a d\right)$, c'est-à-dire les descendants mâles d'alḤariti b. 'Alā', au détriment des femmes tant qu'ils [les descendants mâles] se reproduiraient, et de manière à privilégier les degrés (tabaqāt) [supérieurs] par rapport aux inférieurs. Si les hommes s'éteignent, elle [la $d \bar{a} r$ ] revient aux femmes qui descendent de lui [al- Hāriț] par les reins (mina al-ṣulb). Si les femmes

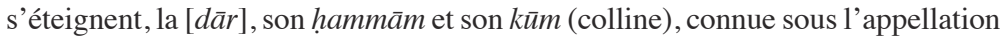
de Abū Qašāšš, seront divisés en trois tiers: un tiers au profit du djihad (fì sabīl Allāh), un tiers pour les pauvres et les indigents, et un tiers au profit de ses clients, les clients de ses enfants et ses enfants tant qu'ils se reproduiront, et ceci après son entretien (maramma) et la paie d'un superviseur (qayyim) si elle en a un. Si les clients s'éteignent et qu'il ne reste plus aucun d'eux, [les revenus seront versés] aux pauvres et indigents de Fusțâț-Mișr et à ceux de Médine ${ }^{13}$.

Si Ibn 'Abd al-Hakam ne recopie pas in extenso l'acte d'immobilisation de la dār al-silsila, il en reproduit sans doute, dans ce passage, les grandes lignes. Les historiens pré-fatimides avaient en effet accès aux documents administratifs et juridiques de la capitale, et les utilisaient comme l'une des sources de leurs informations ${ }^{14}$. La date de fondation $(t a h b i \bar{s} s)$ manque dans le texte, mais il est

12. Sur le processus de la construction de cette demeure, voir J.-C. GARCIN, «Habitat médiéval et histoire urbaine à Fustāt et au Caire», dans J.-C. GARCin, B. MAury, J. Revault, M. ZAKARIYYA éd., Palais et maisons du Caire, vol. I : Époque mamelouke, Paris, 1982, p. 154-155.

13. IBN 'ABD AL-HАKAM, Futūh Mișr..., p. 135-136.

14. Dans un article récent, Wadād al-Qāḍī a démontré ce fait, en analysant un texte d'al-Kindī. Cf. W. AL-QADİ, «An Umayyad Papyrus in the Kindī’s Kitāb al-qud̄at ?», Der Islam, 84 (2007), p. 200245. Voir aussi en ce qui concerne Ibn Yūnus et son utilisation des documents du dīwān al-ğund (les registres militaro-financiers), S. BOUdERBALA, Ğund Mișr : étude de l'administration militaire dans l'Égypte des débuts de l'Islam, Thèse de doctorat, Université de Paris I, 2008, p. 62-65. 
possible de la placer aux alentours de la fin $\mathrm{du} \mathrm{I}^{\mathrm{er}} / \mathrm{VII}^{\mathrm{e}}$ siècle, si l'on se fie aux trois générations de la famille d'Abū 'Abd al-Raḥmān qui ont successivement possédé cette $d \bar{a} r$ jusqu'à son immobilisation par al-Hārit b. 'Alā'. Une immobilisation ancienne donc, antérieure à coup sûr à la décision de Tawba mentionnée plus haut (118/736). Sur quoi nous renseigne-t-elle? D’abord sur les bénéficiaires de l'usufruit de ce complexe foncier dans le quartier central de la ville ${ }^{15}$. Il s'agit d'une immobilisation privée, au profit de la famille du fondateur, mais surtout de ses proches descendants ( $a l-a q$ ' $a d$ ), qui sont bien désignés dans le texte: c'est la descendance mâle qui jouit des bénéfices de ce hubs, avec une exclusion totale de la descendance femelle. Celle-ci ne pourra en bénéficier qu'après l'extinction définitive de toute descendance mâle d'al-Hāritit. Le texte précise clairement que les femmes qui pourront accéder au statut de bénéficiaires doivent descendre d'alHāarit par les reins (al-șulb), ce qui signifie que, si une chaîne de la descendance est coupée par une femme, elle ne sera pas comptée comme bénéficiaire. Nous retrouverons cette problématique dans les autres actes d'immobilisation qui seront analysés plus loin, de même que dans les débats juridiques consignés dans les ouvrages de fiqh se rapportant à l'institution du hubs.

La deuxième information fondamentale est cette clause qui précise les ultimes bénéficiaires de ce bien en cas d'extinction définitive de la descendance rénale - mâle et femelle - d'al-Hāritit. L'usufruit sera divisé en trois tiers comme suit: un tiers pour les clients, mawā $\bar{l}^{16}$, et leurs descendants tant qu'ils se reproduiront ( $m \bar{a}$ tanāsalū $)$, un tiers pour financer le djihad $(f \bar{\imath} s a b \bar{l} l \text { Allāh })^{17}$ et un tiers pour les pauvres et indigents de Fusțāt et de Médine. Outre le fait que cela montre une présence importante de mawālì dans la famille en question, il convient de mettre l'accent sur les deux destinations plus «publiques » et charitables: le djihad d'un côté, les pauvres et les indigents de l'autre. Deux destinations qui sont surtout citées dans le Coran, dans un verset qui constitue le fondement divin de ces fondations. Il s'agit du verset 60 de la sourate al-tawba ( «Le repentir»), qui stipule:

15. Le texte d'Ibn 'Abd al-Hakam fait mention d'une mosquée (masğid) construite à l'intérieur de la dār al-silsila, la mosquée des cornes (al-qurūn), bien connue dans la ville et se trouvant dans le quartier central appelé al-Rāya, l’Étendard: voir ses Futūh Miṣr, p. 135.

16. Les mawālī (sing. mawlā) sont les clients de la société arabe. Institution antéislamique, le walā' évolue en Islam et devient l'institution par excellence par laquelle les convertis rejoignent la société musulmane en se rattachant à un patron dont ils s'attribuèrent la généalogie. Voir P. CRONE, «Mawlā», Encyclopédie de l'Islam, $2^{\mathrm{e}}$ éd., vol. VI (1991), p. 865-866. Pour une mise à jour complète sur cette institution, voir M. Bernards, J. Nawas éd., Patronate and Patronage in Early and Classical Islam, Leyde, 2005.

17. Le terme fì sabīl Allāh signifie littéralement combattre sur le chemin de Dieu. Il est emprunté au Coran (IX/60). Voir C.E. Bosworth, «Sabīl», Encyclopédie de l'Islam, 2e éd., vol. VIII (1995), p. 679. 
Les aumônes ne doivent revenir qu'aux pauvres et aux indigents, à la rétribution de ceux qui s'en chargent, au ralliement des bonnes volontés, à affranchir des nuques (id est : des esclaves), à libérer des insolvables, à [financer le combat] sur le chemin de Dieu (fì sabīl Allāh) et à secourir les enfants du $s a b \bar{l} l^{18}$.

À n'en point douter, ce verset constitue la référence juridique en matière d'aumônes (șadaqāt), dans son sens large, incluant les biens inaliénables. Et c'est probablement sur ce passage coranique que Tawba s'est appuyé pour émettre sa décision d'intégrer la gestion de ces biens dans son administration. Le terme utilisé dans le récit d'al-Kindī pour les désigner est celui de șadaqāt, dont on a vu plus haut qu'il était l'un de ceux qui désignait ce genre de fondations. De même, l'évocation des pauvres et des indigents comme ultimes bénéficiaires n'est pas fortuite. Elle montre le fondement coranique de la décision du cadi. En effet, l'expression «les pauvres et les indigents» (al-fuqarā'wa-l-masākīn) sonne plus comme un concept juridique que comme une catégorie sociale prise en charge par l'État via les aumônes ${ }^{19}$. Cela nous conduit à nous interroger sur l'existence d'une règle, à cette époque précoce, qui contraignait les fondateurs de biens inaliénables à intégrer une dimension charitable dans leurs actes. Le texte relatif à dār al-silsila ne permet pas de l'affirmer, même s'il suppose l'existence d'une clause visant les pauvres et les indigents comme ultimes bénéficiaires. En revanche, la décision de Tawba suppose que les actes de cette période devaient inclure (pour être validés ?) une œuvre de bienfaisance. Pour essayer de mieux cerner la question, il convient de présenter d'autres exemples de cas de hubs à Fusțât et les conséquences judiciaires qu'ils entraînèrent.

Le deuxième exemple concerne le complexe urbain $(d \bar{a} r)$ de Šurayḥa, fille de 'Imrān b. 'Abd al-Raḥmān b. Šuraḥbīl b. Hasana, éminent notable, cadi et chef de la šurța de Fusțāt à l'époque marwānide (86-89/706-708) 20. Selon l'historien mamelouk Ibn Duqmāq (m. 809/1406), Šurayḥa avait immobilisé (habbasat) sa dār au profit des Banū Ḥasana. Ceux-là s'éteignirent en l'an 300/912, et les cadis l'intégrèrent par conséquent dans le financement du djihad (tasallamahā al-quḍat $f \hat{i}-l-s a b \bar{\imath} l)^{21}$. Le texte est court et brut, mais livre tout de même des informations supplémentaires quant à la nature des $a h ̣ b \bar{a} s$ de Fusțāt à l'époque étudiée. Il s'agit bien d'un hubs ancien, puisque sa fondatrice a vécu dans la deuxième moitié du

18. Le Coran, trad. J. Berque, Paris, 1990, IX, 60 (traduction modifiée).

19. Un papyrus arabe du début $\mathrm{du} \mathrm{II}^{\mathrm{e}} / \mathrm{VIII}^{\mathrm{e}}$ siècle confirme l'appellation de cette catégorie (al-fuqarā' wa-l-masākīnn) comme destinataire de l'aumône régulière, la zakāt: G. KHAN, Studies in the Khalili Collection, vol. I: Selected Arabic Papyri, Londres, 1992, p. 49-59 (= P. Khalili I 1). Sur les notions de șadaqa et de zakāt, voir en dernier lieu les analyses de P. SuJPESTEun, Shaping a Muslim State.Papyri Related to a Mid-Eighth-Century Egyptian Official, $\mathrm{PhD}$, Princeton University, 2004, p. 148-159.

20. Sur ce cadi, voir AL-KINDĪ, Kitāb al-wulāt..., p. 326-329.

21. Ibn DuQmāe, al-Intișār li wāsițat 'iqd al-amșār, éd. K. Vollers, Le Caire, 1893, vol. IV, p. 7. 
$\mathrm{I}^{\mathrm{er}} / \mathrm{VII}^{\mathrm{e}}$ siècle, au plus tard au début du II $/ \mathrm{VIII}^{\mathrm{e}}$ siècle. Ce cas est intéressant puisque le fondateur du bien est une femme, et les bénéficiaires sont les descendants de sa famille agnatique, les Banū Hasana; cela veut dire que Šurayha n'avait probablement pas de descendance propre, d'enfants «de ses reins». Malgré le caractère elliptique du texte, il est possible de reconstituer l'acte d'immobilisation de cette $d \bar{a} r$ comme suit: Šurayḥa immobilise sa dār au profit des Banū Ḥasana tant qu'ils se reproduiront. À leur extinction définitive (inqirāọ $)$ ), les bénéfices iront au financement du $s a b \bar{\imath} l$ - c'est-à-dire du djihad. Le transfert du bien dans l'administration judiciaire en l'an 300/912 ne signifie pas que sa gestion était auparavant entre les mains de ses bénéficiaires. La gestion de ces biens dépendait, depuis la décision de Tawba, exclusivement du cadi de la ville. Cela signifie tout simplement que le cadi en fonction en l'an 300/912 prit acte de l'extinction du dernier membre des Banū Ḥasana et transféra l'acte de la dār dans le département judiciaire chargé du financement du djihad. Nous savons en effet que l'entretien des zones frontalières égyptiennes (les mawāhhĭz, pl. de $m \bar{a} h \bar{h} \bar{z}$ ) et le paiement des soldats se faisaient à partir de l'argent des biens inaliénables destinés au sabī ${ }^{22}$. Cela nous amène à nous interroger sur les discussions judiciaires et juridiques concernant la gestion de ces biens à l'époque où les cadis étaient devenus les responsables de l'administration des $a h b \bar{a} \bar{s}$. Nous nous intéresserons dès lors à deux cas de ḥubs datant de l'époque umayyade et qui ont laissé des traces dans les biographies des cadis de la deuxième moitié $\mathrm{du} \mathrm{II}^{\mathrm{e}} / \mathrm{VIII}{ }^{\mathrm{e}}$ siècle.

Le premier cas concerne le hubs d'un certain 'Umayr b. Abī Mudrik al-Hुawlānī, constitué à l'époque umayyade à une date inconnue. Il fit l'objet d'une correspondance d'une extrême importance entre le cadi de Fusțāt, al-Mufaḍal b. Faḍāla ${ }^{23}$, et son maître, Mālik b. Anas. Le contenu de cette correspondance a été conservé par Muḥammad b. al-Rabī ${ }^{4}$ al-Ğ̄īzīi ${ }^{24}$, qui écrit ${ }^{25}$ :

22. Al-Kindī, Kitāb al-wulāt..., p. 418-419, trad. M. TILLIER, Histoire des cadis ..., p. 186-187: «Les zones frontalières (mawāḥ̄z) d'Égypte étaient peuplées par des soldats de l'armée régulière ( $a h l$ $a l-d \bar{\imath} w a \bar{n} n$ ) et des groupes d'engagés volontaires (muțawwi 'a). [Les revenus] des fondations pieuses consacrées [au combat] dans le chemin [d'Allāh] ( $a h b a \bar{s} s$ al-sabīl), gérés par les cadis, étaient collectés chaque année: au mois de juillet $(a b \bar{l} b)$ du calendrier copte, le cadi envoyait chercher l'argent ainsi amassé et le répartissait dans les zones frontalières, depuis al-'Arīš jusqu'à Lūbiyya et Marāqiyya: il était distribué aux engagés volontaires ainsi qu'aux pauvres de l'armée régulière».

23. Deux fois cadi de Fusțāt (168/784-785-169/786, puis 174/790-177/793), grand disciple de Mālik b. Anas, qu'il consultait régulièrement dans les affaires juridiques épineuses et rendait, selon al-Qāộ̄ 'Iyād, « des avis juridiques (fatwā-s) conformes à la doctrine de Mālik». Cf. AL-Q̄̄DI 'IYĀD, Tartīk al-madārik wa taqrīb al-masālik li ma'rifat a 'lām maḍhab Mālik, Beyrouth-Tripoli, 1967, vol. II, p. 456. Sur al-Mufaḍḍal, voir IBn Yūnus, Ta’rīh Ibn Yūnus, vol. I, p. 482-483; AL-KINDī, Kitāb al-wulāt..., p. 377-383, 385-387; trad. M. TiLLIER, Histoire des cadis..., p. 141-146, 149-152.

24. Lettré fustạțien mort en 324/936 et auteur d'un ouvrage intitulé Ahbār qudāt Miṣr, dont quelques passages ont été conservés par Ibn Ḥağar, comme celui que nous entreprenons d'analyser. Sur al-Ğīzīi, voir AL-SAM"ĀNī, al-Ansāb, éd. Hyderabad, vol. II, p. 144.

25. IBN ḤAĞAR, Raf' al-iṣr, p. 439; trad. M. Tillier, Histoire des cadis ..., p. 152, note 643. 
Al-Mufaḍ̣al b. Faḍāla: j'écrivis à Mālik [b. Anas] à propos du bien inaliénable (hubus) de 'Umayr b. Ab̄̄ Mudrik al-Hुawlān̄i. Je lui [en] envoyai la copie conforme et lui écrivis : «Ceux qui ont réclamé que l'immobilisation du bien soit établie $(i t b \bar{a} t)$ sont les descendants des fils ayant entériné la décision de leur père; ils avancent que Hayr b. Nu 'aym leur a écrit [un acte] entérinant l'immobilisation du bien au profit de la descendance masculine des fils, et que les cadis n'ont jusqu'à présent jamais jugé que les filles des fils - ni personne d'autre - ne pouvaient hériter d'une part du bien. Ceux qui prétendent en hériter avancent que leur ancêtre n'a pas mentionné d'ultime bénéficiaire après l'extinction de leur lignée, comme cela se fait dans la transformation d'un bien en fondation pieuse.» [Mālik] me répondit : «J'ai réfléchi au cas du bien inaliénable d'Ibn Abī Mudrik ainsi qu'aux arguments de ceux qui souhaitent en hériter. J'ai trouvé ceci dans l'acte établi par Ibn Abī Mudrik et rapporté par ses descendants, qui l'ont reconnu et entériné : Toutes ses maisons sont immobilisées au profit de ses fils, et un tiers des revenus excédentaires - une fois ses fils logés - ira à la cause de Dieu (fì sabīl Alläh).» Il dit: «Ainsi en va-t-il également pour le moulin.»

C'est là un texte d'une grande valeur historique puisqu'il donne un exemple unique de l'«empirisme» de l'appareil judiciaire face à des cas de hubs primitifs dont les clauses sont loin d'être claires. La complexité du problème et la difficulté de le résoudre poussèrent al-Mufaḍḍal à demander l'avis du grand maître de Médine. De quoi s'agit-il? Si notre lecture est correcte, les plaignants auprès d'al-Mufaḍdal étaient les filles des fils de 'Umayr, exclues de l'usufruit du bien de leur grand-père sous prétexte qu'il n'avait mentionné que les fils des fils. La distinction est nette, dans la lettre d'al-Mufaḍạal, entre walad al-banīn (les mâles descendant des fils) et nisä' al-banīn (les femmes descendant des fils). Il s'agit donc bien d'une plainte «féminine», déposée par les petites-filles de 'Umayr pour s'adjuger une partie de l'usufruit. Quel était leur argument? Elles avançaient que leur grand-père ( $\breve{g} a d d u h u m$ ) n'avait pas dédié son hubs à une œuvre charitable comme cela se faisait généralement dans un acte de hubs. Elles voulaient par conséquent transformer en quelque sorte le hubs en héritage pour pouvoir jouir des bénéfices. Il faut rappeler que l'affaire passa une première fois, selon al-Mufaḍḍal, devant le cadi Hayr b. Nu 'aym al-Haḍramīi ${ }^{26}$, qui confirma par écrit (kitāb) la jouissance exclusive des enfants des fils qui s'opposèrent à la plainte de leurs cousines. L'examen de l'acte par Mālik montre cependant qu'il existait une clause stipulant la donation, après le logement de ses enfants mâles (banīn), d'un tiers des revenus de ce hubs pour le sabīl, c'est-à-dire le djihad. Mālik considéra alors la dār comme un hubs et non un héritage, puisque l'acte comporte cette clause, et élargit son jugement au moulin qui était sans doute inclus dans le bien foncier immobilisé par 'Umayr.

26. Deux fois cadi de Fusțāt (120/738-128/745, puis 133/751-135/753): AL-KInDĪ, Kitāb al-wulāt..., p. 348-352, 355-356; M. Tillier, Histoire des cadis..., p. 104-111 ; 114-116. 
Cette mention du sabīl montre que rares sont les actes de hubs de cette époque qui ne comprenaient pas une clause stipulant la donation d'une partie de l'usufruit, immédiate ou après extinction des bénéficiaires, au profit de la guerre «sur la voie de Dieu », ou encore pour les pauvres et les indigents. Cela semble s'expliquer par la volonté d'empêcher une aliénation du bien par la voie de l'héritage. Les fondateurs de ces biens étaient-ils tenus d'intégrer la dimension charitable dans leurs actes ? Cette clause incontournable était-elle la meilleure garantie pour pérenniser le bien au profit de sa descendance, et empêcher des personnes non désirables (la descendance féminine par le biais de laquelle la famille agnatique se trouve mélangée à d'autres familles) d'hériter du bien ancestral? Nous sommes tentés de le penser, mais l'absence d'arguments plus solides nous empêche d'aller plus loin. Quoi qu'il en soit, il ressort de l'examen de ces deux cas - dār al-silsila et hubs 'Umayr - une forte tendance, dans les actes égyptiens de cette période, à exclure la descendance féminine de la jouissance de l'usufruit de ces biens. Les deux prochains exemples vont nous permettre de mieux étayer ce point.

L'exemple le plus significatif est celui du hubbs de Dār al-fīl, «la demeure de l'éléphant», repère urbain important à Fusțāț. Elle doit son appellation à un épisode daté de l'époque sufyānide (41/61 à 64/683), quand le gouverneur d'Égypte Maslama b. Muhallad al-Anșārī y mit un éléphant destiné à être envoyé en présent au roi des Indes. Nous savons qu'elle appartint par la suite à un mawlā de Maslama, du nom de Abū 'Utaym, qui l'immobilisa selon les termes d'un acte rapporté par al-Kindī dans son livre perdu Kitāb al-mawālì ${ }^{27}$ :

Abū 'Umar [al-Kindī] dit: al-Ḥārit [b. Miskīn] prononça un jugement au sujet de Dār al-F̄̄l, appartenant à Abū 'Uțaym, mawlā de Maslama b. Muhallad. Abū 'Utaym avait constitué cette maison en bien de mainmorte au profit de ses clients ( mawāl̄ $)$ de Fusțāṭ-Miṣr, qu'il avait désignés nommément dans l'acte de fondation de ce bien. Il s'agissait de Ka‘b b. Sulaymān, de Nāṣiḥ, de Yasār, de Rāfi', ainsi que de leurs enfants, de leurs petits-enfants tant qu'ils se reproduiraient, aussi bien hommes que femmes. Lorsque les descendants s'éteignaient, [les revenus de la maison] étaient partagés en deux parts: la première revenait aux pauvres et aux indigents, et la deuxième revenait aux Banū Sā'ida qui habitaient à Mișr, ainsi qu'aux engagés volontaires et aux membres du dīwān appartenant aux $\overline{\mathrm{A}} 1 \mathrm{Ab} \overline{1}$

27. IBN ḤAĞAR, Raf' al-iṣr 'an qudāt Mișr, éd. A.M. 'UMAR, Beyrouth, 1998, p. 123-124; trad. M. Tillier, Vies des cadis de Mișr 237/851-366/976, Le Caire, 2002, p. 49. Le texte est extrait de la notice qu'al-Kindī a consacrée à Abū 'Utyam. Quoiqu'Ibn Ḥağar ne cite pas ici explicitement le Kitāb al-mawāl̄̄ d'al-Kindī, il semble évident de la lui attribuer. Ibn Ḥağar cite généralement al-Kindī comme source de son information, et les détails de l'acte de fondation rapportés ici ne figurent pas dans le Livre des cadis d'al-Kind̄i. Par ailleurs, nous savons que ce dernier a consacré une notice à Abū 'Utaym dans son livre sur les mawālē, comme le rapporte al-Maqrīzī dans al-Mawā'iž wa-li'tibār bi dikr al-hitaț wa-l-ātār, éd. A. F. SAYYID, Londres, vol. III, 2004, p. 535. 
Duğāna - qui formaient un groupe de clients ( 'aṣaba) de Maslama -,à condition que leurs salaires ('ața ') n'atteindraient pas les deux cents, car dans le cas contraire ils n'avaient droit à rien. Si aucun d'entre eux ne se trouvait à Miṣr, la [seconde] part revenait également aux pauvres et aux indigents. La constitution [de cette maison] en bien de mainmorte (al-mahbas) eut lieu en 93/711-712.

Contrairement aux exemples précédents, nous possédons dans cet acte la date de sa fondation (93/711-712), ce qui en fait la plus ancienne attestation datée d'un bien inaliénable à Fusțāṭ. Le fondateur du ḥubs est un mawlā, ce qui est très rare dans la documentation ancienne. Il l'immobilise au profit de ses mawāle , qu'il nomme expressément dans l'acte (Ka‘b b. Sulyamān, Nāṣiḩ, Yasār et Rāfî‘), ainsi que de leurs descendants tant qu'ils se reproduiront. Avec l'extinction définitive de la descendance des personnes mentionnées, les bénéfices de la fondation devaient se répartir en deux parts. L'une était réservée à l'entretien des incontournables pauvres et indigents. La répartition de la deuxième part est plus complexe puisqu'elle concerne deux catégories différentes: les descendants du patron du fondateur, les Banū Sā‘ida, famille de Maslama b. Muhallad

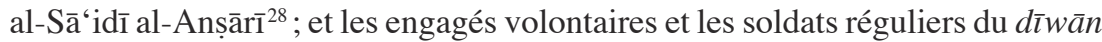
appartenant aux mawālī de Maslama ('aṣaba). Enfin, les mêmes pauvres et indigents récupèreraient la deuxième part des bénéfices (donc, recevraient la totalité des bénéfices) si les deux catégories mentionnées s'éteignaient.

Comme ce fut le cas pour Šurayha, Abū 'Utaym semble ne pas avoir eu de descendance à qui il aurait pu transmettre un bien aussi prestigieux que la $D \bar{a} r$ al-fíl. Il l'immobilisa donc au profit de ses trois clients mentionnés. Le statut du fondateur - un mawlā - domine l'acte de fondation puisqu'une clause inclut la descendance rénale de son patron, Maslama b. Muhallad, comme un geste de reconnaissance à l'égard de l'illustre gouverneur d'Égypte.

L'aspect charitable du bien ne diffère guère des cas exposés plus haut : les pauvres et indigents passent pour être incontournables dans ces biens ahlī des débuts de l'Islam, mais aussi l'entretien des volontaires et des soldats réguliers pauvres qui combattent sur la voie de Dieu, comme le précise le texte d'al-Kindī relatif à l'entretien des $m \bar{a} h \bar{u} z$-s égyptiens par l'argent du sabīl. En revanche, ce qui diffère sensiblement entre ces actes, d'après les échantillons encore conservés, est la question du droit de la descendance féminine à l'usufruit de ces fondations. Si l'acte de la dār al-silsila exclut sans ambigüité les femmes des bénéfices de la fondation (les descendants mâles au détriment de la descendance féminine), celui de dār al-fíl garantit, en apparence, une égalité entre les deux sexes (aussi bien hommes que femmes). Cette question a été l'un des points de désaccord les plus souvent mentionnés entre les doctrines mālikite et ḥanafite en matière de biens 
inaliénables. C'est aussi une question de pratique sociale, qui se manifeste par ailleurs d'une façon éclairante dans le plus ancien document de hubs de l'Islam conservé, rédigé dans le Fayyūm au début du III $/ \mathrm{IX}^{\mathrm{e}}$ siècle ${ }^{29}$.

Ce document et les bribes rapportées par al-Kindī permettent d'éclairer quelque peu la compétition entre les écoles mālikite et hanafite autour d'une question sociale relative à l'héritage par le biais de ces biens.

En effet, le hubs de Dār al-fill, constitué donc en 93/711-712, a retenu l'attention des cadis de la ville pendant plus d'un siècle, autour du droit des descendants des femmes à bénéficier de l'usufruit de la fondation. L'affaire passa devant six cadis, le premier étant Tawba b. Namir, ce qui semble logique puisqu'il fut le premier à avoir intégré la gestion des biens inaliénables au sein de l'administration judiciaire. Un certain Wattăğ, mawlā de Abū 'Utaym et résident en Ifrīqiya, vint réclamer son droit comme bénéficiaire du hubs auprès de Tawba, qui refusa sa demande en arguant qu'il ne figurait pas dans l'acte d'immobilisation. En 117/735-736, Tawba prononça donc une sentence au profit des enfants des nommés qui étaient présents à l'audience.

L'affaire prit ensuite une plus grande ampleur quand les cadis de Fusțāt furent amenés à juger le droit des descendants de 'Azza b. 'Amr, petite-fille de Rāif', mentionné dans l'acte de fondation. Le premier cadi à avoir donné un jugement sur la question est al-Mufaḍḍal b. Faḍāla (174-177/790-793). À l'époque d'al-Mufaḍḍal, il ne restait plus, de la descendance des bénéficiaires nommément cités dans l'acte, que Muhammad b. Nāṣị̣ et 'Azza b. 'Amr b. Rāfi'. À la mort de cette dernière, son fils, Ibrāhīm b. 'Abd al-Șamad b. al-Sā'ih, demanda au cadi al-Mufaḍdal de lui attribuer la part de sa mère. Le cadi refusa et remit l'ensemble des bénéfices du huubs à Muhammad b. Nāṣiḥ ${ }^{30}$. En faisant cela, al-Mufaḍdal excluait la descendance des femmes des bénéfices, puisque Ibrāhīm ne descendait pas «des reins » (al-șulb) des personnes mentionnées dans l'acte de fondation, mais d'une personne extérieure, en l'occurrence son père 'Abd al-Șamad. Cette décision allait être confirmée par un autre cadi de tendance mālikite, 'Abd al-Rạ̣mān al-'Umarī (185-194/801-810), avant d'être abrogée par le cadi Ibrāhīm b. al-Ğarrāḥ (205-211/820-826), d'obédience ḥanafite. Après avoir été examinée par six cadis, ainsi que nous l'avons dit, l'affaire fut résolue au milieu du $\mathrm{III}^{\mathrm{e}} / \mathrm{IX}^{\mathrm{e}}$ siècle à la suite de l'intervention personnelle du grand cadi

29. Il s'agit du P. Marchands I 12, un brouillon de hubs privé de la famille des Banū 'Abd al-Mu'min, mise en lumière par Yūsuf Rāgib qui a rassemblé son archive à travers les collections des papyrus arabes éparpillés dans le monde. Selon lui, le document est le plus ancien acte de waqf qui nous soit parvenu de l'Islam. Cela n'empêche pas de considérer les échantillons conservés par al-Kindī comme les plus anciens témoins de rédaction des actes de hubs.

30. IBN ḤaĞAR, Raf‘ al-ișr, p. 124; M. TILlen (trad.), Vie des cadis..., p. 50. 
de Bagdad, qui confirma le droit de la descendance de 'Azza aux bénéfices du hubs de Dār al-fìl et leur en accorda la moitié ${ }^{31}$.

L'enjeu de cette compétition juridique était de savoir si les descendants des femmes avaient droit à l'usufruit du hubs. La question est d'importance capitale puisqu'elle vise à les exclure de leur héritage inaliénable dans un cas (vision mālikite), et à leur en reconnaître le droit dans l'autre (vision hanafite). Cette compétition dénote aussi une volonté du pouvoir central d'intervenir directement dans la gestion de ces biens en envoyant en Égypte des cadis représentant le $m a \underline{d} h a b^{32}$, qui tendait à s'affirmer comme la doctrine officielle de l'Empire, le ḥanafisme. À Fusțạt, le premier cadi de tendance hanafite, nommé directement par le calife al-Mahdī en 164/780, fut Ismā̄īl b. Alīsa' al-Kindī. Il fut bien accepté par les élites de la ville, sauf en matière de gestion des biens inaliénables. Voici comment un lettré fusțātien décrit l'exercice de la judicature par Ismā ‘̄îl ${ }^{33}$ :

Sa‘īd b. Abī Maryam: Ismā‘̂̄l b. Alīsa‘ al-Kindī nous arriva en tant que cadi à la révocation d'Ibn Lahī'a. Il fut l'un de nos meilleurs cadis, bien qu'il ait adhéré à la doctrine (madhab) d'Abū Hanīfa. Les Égyptiens ne le reconnaissaient pas et le prirent en grippe. Sa doctrine préconisait l'abrogation des fondations pieuses $(a h b a \bar{a} s)$; cela pesait trop aux Égyptiens et ils le prirent en grippe.

C'est dire tout l'enjeu socio-économique que ces biens inaliénables de Fusțāt représentaient pour le pouvoir central. Engagé dans une guerre de longue haleine avec l'Empire byzantin, le pouvoir abbasside, sous al-Mahdī (r. 158-169/775-785) et Hārūn al-Rašīd (786-809), devait trouver des financements importants pour entretenir les volontaires et les soldats réguliers au front. C'est probablement de là que vient la volonté de l'appareil judiciaire de consacrer une partie des bénéfices des $a h b \bar{a} s$ de Fustạt au sabīl. Nous avons vu que les $m \bar{a} h \grave{u} \bar{z}$-s égyptiens étaient essentiellement entretenus par l'argent provenant des biens inaliénables, sans que l'on sache exactement de quand date cette mesure. La nomination d'Ismā'îl en 164/780 pourrait être la date à partir de laquelle les cadis mirent en œuvre cette politique, puisque la doctrine hanafite est plus exigeante en matière de loi sur les successions par la fondation des biens de mainmorte ${ }^{34}$. Et c'est probablement pour cette raison que l'élite de la ville s'opposa farouchement aux nouvelles

31. Pour les détails de cette histoire, voir AL-KInDĪ, Kitāb al-wulāt, p. 474-475 ; IBN ḤAĞAR, Raf' al-ișr..., p. 124 ; M. Tillier (trad.), Vies des cadis..., p. 50-51.

32. C'est le terme que préfèrent utiliser les spécialistes de l'histoire juridique des débuts de l'islam, selon lesquels à cette époque (deuxième moitié $\mathrm{du} \mathrm{II}^{\mathrm{e}} / \mathrm{vIII}^{\mathrm{e}}$ siècle), on ne peut pas vraiment parler d'école hanafite bien établie. Voir la mise au point de M. TilLier, Les cadis d'Iraq et l'État Abbasside (132/750-334/945), Damas, 2009, p. 151-157.

33. Al-Kindī, Kitāb al-wulāt, p. 371; trad. M. Tillier, Histoire des cadis..., p. 134.

34. Abū Hanīfa considérait que seuls les descendants procréés au moment de la mort du fondateur pouvaient bénéficier de l'usufruit d'un bien inaliénable ahlī et qu'à leur mort, tous les 
mesures prises par ce cadi irakien. Al-Kindī rapporte un débat qui aurait eu lieu entre Ismā‘îl et al-Layt b. Sa'd, le plus éminent des juristes de la ville, autour de la nouvelle politique du cadi concernant les aḥbās de Fustạat. Selon le même auteur, Ismā‘îl se vit révoquer par le calife al-Mahdī à la suite de ce débat ${ }^{35}$.

Le débat déclenché par l'affaire de dār al-fìl s'inscrit, croyons-nous, dans cette compétition entre une tradition égyptienne ancienne, de plus en plus influencée par l'école mālikite, et une doctrine hạanafite irakienne élaborée autour d'Abū Hanīfa et ses deux grands disciples, al-Šaybān̄̄ (m. 189/805) et Abū Yūsuf (m. 182/798). Nous avons vu, d'après les deux exemples avancés - dār al-silsila et $d \bar{a} r$ al-fíl-, que les fondateurs d'un bien inaliénable privé avaient l'entière liberté de nommer les bénéficiaires dans leurs actes. Ce n'est qu'au moment où les juristes s'accaparèrent du dossier de ces biens que l'on commença à établir des règles juridiques de nomination des bénéficiaires. L'école hịāzienne semble avoir été hostile au droit des femmes de jouir des bénéfices des aḥbās, comme le montre ce passage de la Mudawwana, attribué à Mālik b. Anas ${ }^{36}$ :

Rien ne revient aux descendants des filles si l'homme [i.e. le constituant] dit: cette maison est un hubs au profit de ma descendance (waladī). Elle revient par conséquent à ses fils et aux descendants de ses fils, et rien ne revient aux descendants des filles.

La tradition hị̆gāzienne archaïsante prône une vision agnatique de la jouissance de l'usufruit du hubs, seuls les descendants des mâles ayant accès aux bénéfices de la fondation. En revanche, ceux qui ne descendent pas des « reins» du fondateur, c'est-à-dire les enfants descendant d'une femme, en sont exclus. La raison est à chercher dans la volonté des juristes «d'écarter les enfants d'étrangers afin d'éviter la désagrégation et le morcellement du patrimoine et d'assurer sa conservation à la famille dans le sens strict du mot, à savoir le groupement fondé sur la parenté mâle ${ }^{37} \gg$. Il semble aussi qu'un débat s'était instauré à Médine au début $\mathrm{du} \mathrm{II}^{\mathrm{e}} / \mathrm{VIII} \mathrm{e}^{\mathrm{e}}$ siècle pour savoir si les femmes «descendant des reins » du fondateur devaient être exclues comme bénéficiaires. Ce débat reflète sans aucun doute des pratiques sociales de succession qui éliminaient de fait les femmes de ce droit, comme le montre le cas de dār al-silsila où il est dit explicitement «au profit de la descendance mâle d'al-Ḥaritit, au détriment des femmes ». Ce débat

revenus devaient revenir aux indigents. Voir AL-HुASSĀF, Ahkām al-waqf..., p. 94; P. Hennigan, The Birth of a Legal Institution..., p. 96-101; M. TILLIER, Histoire des cadis..., p. 134, note 581.

35. Al-KInDī, Kitāb al-wulāt..., p. 372; M. TILlIER, Histoire des cadis..., p. 135.

36. SAhnun в. SA 'īD, al-Mudawwana al-Kubrā,p. 421. Toute la théorie mālikite sur l'exclusion des descendants des filles est fondée sur l'interprétation du verset 11 de la sourate Iv, relatif à l'héritage et à la part des mâles par rapport aux femelles. Cet avis juridique est mieux exposé dans Ibn Abī Zayd, al-Nawādir wa-l-ziyādāt, éd. A. HูATṬ̄̄BĪ et M. DABBĀĞ, Beyrouth, vol. XII, 1999, p. 24-25.

37. Y. RĀĞIB, Marchands d'étoffes..., p. 39 et note 5. 
remonte, selon les sources juridiques, au califat de 'Umar b. 'Abd al-'Azīz (99101/717-720), qui aurait voulu consacrer par une loi l'exclusion des femmes. Les juristes du Hiğāa se seraient alors appuyés sur une tradition de ' $\bar{A}$ ' išă ${ }^{38}$ dans laquelle elle condamne la privation des femmes des bénéfices du hubbs, en comparant cette action à une pratique mécréante réprouvée par le Coran ${ }^{39}$. En revanche, la tradition irakienne dominée par les premiers hanafites reconnaît le droit des femmes à l'usufruit des biens inaliénables privés, comme le montre la réponse d'al-Šaybān̄̄ aux lettrés de Médine ${ }^{40}$ :

Celui qui légalise le ḥubs en disant «ma demeure est un huubs au profit de mes enfants et des enfants de mes enfants », doit inclure les enfants des femmes dans [la catégorie] des enfants de ses enfants parce que la fille fait partie de ses enfants. Par conséquent, ses enfants (i.e. de la femme) font partie des enfants de ses enfants (i.e. du fondateur).

Il s'agissait donc de savoir si les descendants des femmes devaient jouir ou non de l'usufruit de ces biens. La doctrine hanafite, considérée comme plus «libérale» et «égalitaire», consacrait l'égalité entre les deux sexes ${ }^{41}$, ce que confirment d'ailleurs les différentes interventions des cadis hanafites de Fusțāt que nous avons évoquées : ils proposent de donner la moitié (donc à part égale) des bénéfices de $d \bar{a} r$ al-fĭl aux descendants de 'Azza, alors que les cadis mālikites les en ont exclus. Ceci montre qu'au moment de la rédaction de ces actes, la norme juridique concernant les bénéficiaires n'existait pas encore et que les fondateurs avaient libre choix de désigner les descendants ayant accès à l'usufruit. Sans doute s'appuyait-on sur la Coutume, le 'urf, qui devait varier d'un milieu à l'autre et d'une ville à l'autre.

Assurément, la période qui s'étend de la fin $\mathrm{du} \mathrm{II}^{\mathrm{e}} / \mathrm{VIII}^{\mathrm{e}}$ siècle au début du III/IX siècle a vu s'exprimer une compétition juridique autour de la question des biens inaliénables, sans qu'une norme juridique ait pu s'imposer définitivement. Nous pouvons d'ailleurs en prendre pour preuve le brouillon de l'acte de hubs des

38. 'Ā'iša, fille du premier calife bien guidé, Abū Bakr, et femme préférée de Muhammad. Elle se distingua essentiellement par son rôle politique lors des événements de la première guerre civile, mais aussi dans la transmission de quelques traditions prophétiques. Voir W.M. WATT, «'A' isha», Encyclopédie de l'Islam, 2e éd., Leyde, vol. I (1986), p. 307-308.

39. Iвn Aвī ZAYD, al-Nawādir wa al-ziyādāt, vol. XII, p. 7, sous le titre: «De la réprobation d'exclure les femmes [de l'usufruit] du hubs». Le verset en question est le VI/139: «Ce que ces bêtes de troupeau ont dans le ventre est voué à nos mâles, interdits à nos femelles » (traduction $\mathrm{J}$. Berque, modifiée).

40. Al-ŠS AYBĀNī, al-Huğğa 'alā ahl al-Madīna, vol. III, p. 56.

41. Al-HुAssāF, Ahkam al-awqäf..., p. 28. 
archives des Banū 'Abd al-Mu'min, publié et étudié de très près par Yūsuf Rāgib. Nous reproduisons ici les clauses qui touchent directement à notre analyse ${ }^{42}$ :

[...] que la maison qu'ils possèdent en tel endroit, dont les limites sont telles, est une immobilisation en donation aumônière (hubs șadaqa) spécialement affectée à Ğa'far, Tāabit et Raysān, aux enfants de Ğa'far, de Tāabit et de Raysān, et aux enfants de leurs enfants tant qu'ils se reproduiront et à tous ceux dont la filiation remonte aux reins d'Ahmad b. 'Abd al-Mu'min. Les mâles et les filles dans le partage seront égaux... Rien ne reviendra aux enfants des filles dans cette immobilisation, qu'à la fille dont la filiation remonte aux reins d'Aḥmad b. 'Abd al-Mu'min.

Yūsuf Rāgib suggère que cet acte fut rédigé selon le madhab hanafite, en prenant comme preuve la clause qui désigne les fondateurs du hubs comme bénéficiaires immédiats de l'usufruit, dans le sens où l'on fait donation en faveur de soi-même ${ }^{43}$. Pourtant, plusieurs éléments permettent de douter du caractère hanafite de cet acte, que nous plaçons plutôt sous la juridiction mālikite à laquelle appartenait la famille des Banū 'Abd al-Mu'min ${ }^{44}$. Yūsuf Rāgib avance que «les premiers juristes étaient unanimes à déclarer que la condition essentielle de la validité du waqf était le dessaisissement immédiat du constituant au profit du bénéficiaire». S'écartant de la doctrine du maître Abū Hanīfa, Abū Yūsuf vint à déclarer que le constituant ne devait pas obligatoirement se dépouiller de son vivant, avis qui triompha dans le rite hanafite malgré la réticence et l'opposition de plusieurs juristes ${ }^{45}$. Il est vrai que seule la doctrine hanafite permet au donateur de devenir donataire, mais cela dérive sans doute d'une pratique sociale qui a dû influencer la constitution d'une telle norme. Nous n'en savons rien en ce qui concerne l'Irak, puisque nous ne possédons aucune trace réelle d'un acte de hubs primitif. En revanche, un papyrus daté de 102/720 atteste une telle pratique en Égypte. Il s'agit d'un testament rédigé par une femme à bord d'un bateau. Se trouvant en danger de mort, elle décide d'affranchir son esclave (dont le sexe n'est pas connu) et d'immobiliser sa maison et son vignoble dans les termes suivants :

J'ai constitué la maison et le vignoble en bien de mainmorte (habīs), invendable pour que tu puisses y habiter après [ma mort], et pour que je puisse y habiter tant

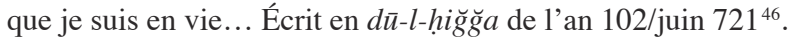

42. P. Marchands I 12, 1. 3-10.

43. Y. RĀ̇̈IB, Marchands d'étoffe..., p. 37.

44. C'est l'examen même des documents de cette famille (contrat de société, des obligations en tissu) qui permet à Y. Rā̄ỉB (Marchands d'étoffe..., p. 37-38) d'affirmer leur appartenance au madhab mālikite.

45. Ibid, p. 37, en se fondant sur les deux juristes irakiens al-Hasșāâf et Hilāl.

46. P. Ragab 6. 
Malgré l'état fragmentaire du document et l'absence des principales clauses concernant cette décision, l'essentiel est là : la maison et le vignoble sont immobilisés au profit de l'esclave affranchi, mais aussi au profit de la donatrice qui se constitue donataire. Et l'on ne peut s'empêcher de se demander si cette pratique ne fut pas assez courante à cette époque, ce qui expliquerait que les juristes aient eu à condamner de telles pratiques et à décréter la non-validation d'un huubs si le donateur était lui-même bénéficiaire.

Mais cela ne constitue pas la raison essentielle qui nous pousse à douter du caractère hanafite de cet acte. L'on a vu que seul le madhab hanafite permet aux descendants de filles d'être bénéficiaires d'une donation. Or il est bien stipulé que les bénéficiaires de la donation doivent recevoir une part égale, les mâles comme les femmes, mais que les enfants des femmes doivent en être exclus («Rien ne reviendra aux enfants des filles dans cette immobilisation»). Cette clause spécifique montre que l'acte obéit au madhab mālikite, puisqu'il refuse de reconnaître les enfants des filles comme bénéficiaires, contrairement aux hanafites, comme nous l'avons montré dans le cas du hubs de dār al-fìl.

Mais peut-on vraiment parler d'un acte rédigé selon un rite plutôt qu'un autre? Une autre clause vient encore brouiller les pistes : elle concerne Raysān, la sœur des fondateurs et elle-même fondatrice. Au moment de la rédaction de l'acte, elle n'avait pas donné d'enfants à son mari Aḥmad b. Muḥammad b. 'Ayyāš. Le papyrus conservé précise qu'au cas où elle aurait des enfants, ils toucheraient, après le décès de leur mère, trois dinars annuels du loyer de sa maison, puis que ses petits-enfants seraient ensuite définitivement exclus de l'usufruit. Si elle mourait sans donner de progéniture, les trois dinars iraient à son mari (sa vie durant), puis reviendraient, à sa mort, aux frères de Raysān et à leurs descendants ${ }^{47}$.

Autant dire que l'acte dénote un mélange hétéroclite de pratiques sociales touchant essentiellement à la volonté des fondateurs, et de normes juridiques encore très mal implantées. Il nous semble qu'à cette époque les fondateurs des biens inaliénables, vivant dans la campagne de surcroît, avaient une certaine liberté dans la constitution de leurs biens privés. Toutefois, la clause mentionnant l'exclusion des enfants des filles montre clairement que cet acte ne peut être considéré comme émanant d'une vision hanafite, si l'on tient compte des tentatives des cadis hananafites de Fusțāt d'imposer l'accès à la jouissance des biens inaliénables privés aux enfants des filles. 
Les différents cas examinés montrent comment les premiers ḥubs de Fusțât étaient constitués sans fondement juridique, au profit de la famille du fondateur pour, semble-t-il, contourner le droit de succession contraignant. Ils montrent aussi la présence de pratiques différentes, conditionnées probablement par le milieu social ou l'héritage de la Coutume, le 'urf, qui reste un domaine encore inconnu ${ }^{48}$. L'intervention de l'appareil judiciaire à partir de 118/736 est sans doute liée à cette volonté du pouvoir public de mieux contrôler les biens fonciers de la ville, mais aussi de mettre la main sur la gestion d'un patrimoine qui produisait une masse considérable de richesses. La présence de la clause mentionnant les pauvres et les indigents et le financement du djihad ( $f \grave{i}$ sabīl Alläh) dans les actes étudiés confirme leur rattachement à l'enseignement coranique, via le verset 60 de la sourate Ix. Elle montre aussi le souci du pouvoir public de limiter le contournement des bénéfices de ces biens par les familles fondatrices et de leur imposer en quelque sorte une règle contraignante qui stipulait le but ultime de toute fondation pieuse, à savoir la charité dans son sens large. C'est l'essence même de la réforme de Tawba, qui soustrait la gestion de ces biens des mains des bénéficiaires pour en assurer personnellement, en tant que pouvoir judiciaire, le contrôle.

La décision du premier cadi hanafite de la ville, en 164/780, de réformer le système des $a h ̣ b a \bar{s}$ est liée à la volonté du pouvoir 'abbāside, depuis Bagdad, de limiter, dans la durée, la jouissance des descendants du fondateur des bénéfices du bien, selon la doctrine de Abū Hanīfa. Ce dernier considérait en effet que seuls les enfants procréés au moment de l'immobilisation avaient le droit de l'usufruit du bien et que, à leur extinction, tous les revenus devaient revenir aux indigents et au sabīl (c'est-à-dire au djihad) ${ }^{49}$. C'est sans doute pour cette raison que l'élite de Fusțât (intellectuelle, mais aussi sociale) refusa d'y adhérer: une telle mesure remettait en cause la prospérité de nombreuses familles de la ville. L'avis de Abū Ḥanīfa d'accélérer, en quelque sorte, l'accès de l'État aux bénéfices de ces biens (en les distribuant aux indigents, mais surtout en les injectant dans les coûteuses entreprises d'entretien des zones frontalières), était probablement conditionné par le besoin de l'État 'abbāside en ressources matérielles - les biens inaliénables représentaient une manne importante dont il ne pouvait se passer. C'est peut-être aussi pour cette raison que Hilāl al-Ray', mais surtout le grand cadi al-Huașsāaf, rédigèrent leurs traités à cette période, comme pour établir définitivement la politique impériale en matière de gestion de ce qu'on appelle désormais le waqf, terme canonisé par ces deux traités ${ }^{50}$.

48. Y. R̄̄ìiB, Marchands d'étoffe..., p. 39.

49. Al-HुAssĀF, Ahkām al-awqāf..., p. 49; M. TILliER, Histoire des cadis..., p. 134, note 581.

50. Les deux traités sont dépourvus d'introduction susceptible de nous éclairer sur les raisons de leur rédaction - l'on ne sait, dès lors, s'ils ont ou non été commandés par le pouvoir impérial. En 
Les exemples de dār al-silsila, de dār Šurayḥa, du hubs de Abū Mudrik al-Hुawlānī et de dār al-fĭl, jumelés à ceux du testament daté de 102/720 et du brouillon du hubs des Banū 'Abd al-Mu'min, montrent une grande diversité dans la réalisation de cette pratique sociale qu'est le hubs dans l'Égypte des deux premiers siècles de l'Islam. Ces pratiques ont dû être à la base des premières législations locales en matière de hubs, et l'on peut se poser la question de savoir s'il n'existait pas une doctrine «égyptienne» en matière de gestion du $h u b s$, avant que les mad hab-s mālikite et hanafite ne l'emportent à Fusțât ț ${ }^{51}$. Les différentes interventions des cadis de Fustạtat, qu'ils fussent d'obédience mālikite ou hanafite, traduisent la volonté d'un pouvoir public, par le biais de sa judicature, de «normaliser» des pratiques sociales en se fondant sur le Coran et les pratiques et dires supposés des Compagnons. C'était une façon de rompre avec des pratiques issues du 'urf, qui tendaient à contourner la loi de l'héritage mais aussi à assurer la pérennité des biens privés et les éloigner de la mainmise de l'État. Peut-on dès lors parler de rupture? La question est plus complexe: l'observation portée sur plusieurs décennies montre que la longévité des usages se repère encore au moment où l'appareil judiciaire commence à mettre la main sur la gestion de ces biens, et ce n'est que par une forme d'interaction entre la pratique et la théorie qu'un contrôle effectif a pu être possible ${ }^{52}$.

Sobhi Bouderbala - Institut français d'archéologie orientale, Le Caire sbouderbala@ifao.egnet.net

revanche, il n'est pas anodin qu'al-Ḩașșāf, grand cadi de Bagdad, écrivit son traité à un moment où ce poste était étroitement lié au calife, une sorte de «façade légale du califat» selon l'expression de M. Tillier, Les Cadis d'Iraq ..., p. 543 et sq.

51. J. Sснаснт, Introduction, p. 35, avance que l'Égypte ne vit pas se développer d' «ancienne» école juridique régionale au $\mathrm{II}^{\mathrm{e}} / \mathrm{VIII}^{\mathrm{e}}$ siècle. Cette hypothèse a été nuancée récemment par M. TILLIER, «Les premiers cadis... », p. 214-218, où il démontre l'existence d'un milieu savant à Fusțāt, plus ou moins lié à l'école médinoise.

52. Voir sur ce point, pour le cas des contrats de métayage par exemple, les analyses judicieuses de B. JohANSEn, «Coutumes locales et coutumes universelles aux sources des règles juridiques en Droit musulman hanéfite», Annales Islamologiques, 27 (1993), p. 31-34. Voir aussi les analyses de S. Denoix, «Introduction: formes juridiques, enjeux sociaux et stratégies foncières », Revue du monde musulman et de la Méditerranée, 79/80 (1996), p. 11-14; B. DuPRET, «L'historicité de la norme. Du positivisme de l'islamologie juridique à l'anthropologie de la norme islamique », Annales. Histoire, sciences sociales, 54/1 (1999), p. 175-178. 


\section{Les $a h b \bar{b} \bar{a}$ de Fusțāt aux deux premiers siècles de l'Hégire : entre pratiques socio-économiques et normalisation juridique}

Cette étude porte sur les pratiques sociales en matière de biens inaliénables, le hubs (pl. ahbāas), au début de l'Islam, et leur rapport avec les théories juridiques naissantes en se fondant sur une documentation exclusivement égyptienne. Dans un premier temps, nous examinons les spécimens des actes de ces biens parvenus jusqu'à nous, conservés dans la littérature pré-fatimide de Fusțāt, en mettant l'accent sur les pratiques sociales en vigueur à Fusțât dès la fin du $\mathrm{I}^{\mathrm{er}} / \mathrm{VII}^{\mathrm{e}}$ siècle et les spécificités des clauses composant ces actes. Les tentatives des fondateurs de ces biens pour pérenniser leur patrimoine et contourner certaines règles rigides de l'héritage en sont un exemple. Ensuite, l'accent est mis sur l'intervention du corps judiciaire et juridiques (cadis et jurisconsultes) dans la réglementation et la résolution de ces biens à partir du milieu $\mathrm{du} \mathrm{II}^{\mathrm{e}} / \mathrm{VIII}^{\mathrm{e}}$ siècle, en se fondant sur quelques documents papyrologiques des trois premiers siècles de l'Hégire. Cette enquête permet de mieux apprécier l'évolution de l'institution des biens inaliénables en Égypte dans une dynamique de transition qui aboutit, à terme, à normaliser les pratiques sociales en la matière.

hubs - Fusțāṭ - sabīl - cadi - hanafite - mālikite - descendance.

\section{Between Socio-economic Practices and Legal Normalization : The ahbās of Fusțāt during the First Two Centuries of Hegira}

This article focuses on social practices related to endowments, $h u b s$ (pl. $a h b \bar{a} s$ ), during the first two centuries of Islam, and their relations to the legal theories which emerged during that period. The study is based on exclusively Egyptian documentation. We will first examine documents of $a h b \bar{a} s$ from the end of the $1 \mathrm{st} / 7$ th century, which are available in the pre-fatimid literature produced in Fustạt, with a specific focus on social practices and the specific conditions which were stated in these documents. How holders of $a h b \bar{a} s$ managed to make them durable and circumvent some of the strict regulations of inheritance. Secondly, we will look at the part that the cadis and jurisconsults played in regulating and resolving conflicts related to ahbās, from mid 2nd/8th century, based on papyrus documents, from the first three centuries of Hegira. This study is aimed to better comprehend the evolution of the institution of endowments in Egypt, in a context of transition which resulted in normalising social practices in this field. ḥubs - Fusțāṭ - sabīl - Qadi - ḥanafite - mālichite - descendence. 
